\title{
Protein unfolding in freeze frames: intermediates of ubiquitin and lysozyme revealed by variable temperature ion mobility-mass spectrometry.
}

\author{
Jakub Ujma, ${ }^{\ddagger}$ Jacquelyn Jhingree, Rosie Upton, Florian Benoit, Bruno Bellina and Perdita Barran* \\ ${ }^{\dagger}$ Michael Barber Centre for Collaborative Mass Spectrometry \\ Manchester Institute of Biotechnology, University of Manchester \\ 131 Princess Street, M1 7 DN, Manchester, United Kingdom \\ ${ }^{\ddagger}$ Current address: Waters Corporation, Stamford Avenue, Altrincham Road, Wilmslow, SK9 4AX, UK
}

\section{Supporting Information}

The gas phase is an idealized laboratory for the study of protein structure, from which it is possible to examine stable and transient forms of mass selected ions in the absence of bulk solvent. With ion mobility-mass spectrometry (IM-MS) apparatus built to operate at both cryogenic and elevated temperatures, we have examined the conformational transitions of ubiquitin and lysozyme ions as a function of temperature. Following collision activation of the protein ions in the source, the instrument enables subsequent "freezing" or thermal annealing of unfolding intermediates ensemble and measurement of their collision cross sections (CCSs). Data from ubiquitin suggests that the unfolding transitions proceed through diverse and highly elongated intermediate states, which then converge to more compact structures. These findings contrast with data obtained from lysozyme a protein where (un)-folding plasticity is restricted by four disulfide linkages. We show with cold IM-MS experiments how ubiquitin can explore a wide conformational landscape due to its unrestricted nature, whereas for lysozyme the conformational restriction does not allow substantial rearrangement and facilitates enhanced resolution of the native fold as the buffer gas temperature is reduced.

Ubiquitin is a small, highly conserved protein with a native state noted for its stability under a range of solution conditions. ${ }^{1,2}$ Folds of ubiquitin have been comprehensively studied with a variety of experimental methods including NMR, crystallography and also computationally. ${ }^{3-6}$ In solution, ubiquitin exhibits two-state kinetics with a compact native $\mathrm{N}$-state and a partially folded A-state, although, some studies have suggested the presence of additional on- or off-pathway intermediate states. ${ }^{7,8}$ Highly reversible, conformational transitions have been induced by altering solution conditions, ${ }^{9-11}$ pressure $^{12}$ and temperature. ${ }^{11,13}$ Time-resolved $\mathrm{NMR}^{7}$ has been used to probe transitions on a several seconds timescale, and time-resolved IR experiments have shown intermediate states occurring on a micro to millisecond timescale. ${ }^{8}$ The intrinsic characteristic of either spectroscopic approach is the spatially averaged nature of observables. Whilst time-resolved information is indispensable for kinetic analyses, inferring the intermediates' global geometrical structure solely from spectral data of a solvated ensemble is not straightforward. The gas-phase technique of ion mobility-mass spectrometry (IMMS) allows measurements of a so-called collision cross section (CCS) on a millisecond timescale. In the crudest hard sphere approximation, the CCS parameter can be seen as a rotationally averaged shadow projection of a protein conformation. ${ }^{14}$ When correlated with the ion abundance, a CCS distribution yields intuitively interpretable information about the conformational preferences of a molecule. Using IM-MS, several groups have investigated conformational preferences of gaseous ubiquitin ions at ambient temperatures. ${ }^{15-19}$ Typically, in the absence of solution, ubiquitin ions generated from "native" solution conditions have been found to populate compact conformational states with a ${ }^{\mathrm{DT}} \mathrm{CCS}_{\mathrm{He}}$ of around $950 \AA^{2}$. This compact state has been assigned as the N-state also observed by NMR and crystallography. ${ }^{19}$ Structural changes of the compact state have been observed after prolonged residence times inside the vacuum, upon collisional activation, as a function of charge state and following thermal denaturation in solution. ${ }^{17-22}$ It has been observed that under such conditions, the protein adopts intermediate I-states (with ${ }^{\mathrm{DT}} \mathrm{CCS}_{\mathrm{He}}$ 1400-1700 $\AA^{2}$ ) which are also correlated with a solution-phase specific A-state. ${ }^{19}$ Extended structures with a ${ }^{\mathrm{DT}} \mathrm{CCS}_{\mathrm{He}}$ of up to $2000 \AA^{2}$ have also been reported and classified as gas-phase specific conformations or the unfolded U-state. ${ }^{16,17,19}$ Notably, Clemmer and Jarrold were the first to show that both increased temperature of the buffer gas and collisional activation can lead to elongation events, ${ }^{23}$ attributed to unfolding pathways. ${ }^{24}$ Similarly, gas phase compaction following charge reduction has been demonstrated by Valentine et al. $^{25}$ and more recently by Laszlo et $a l{ }^{26}$ These elongation/compaction phenomena appear substantially "step-wise"; conceptually resembling mechanically induced unfolding experiments performed by atomic force microscopy. ${ }^{27}$ Brueker et al. investigated ubiquitin ions using ion heating followed by electron capture dissociation (ECD). The general "threestate" unfolding model has been verified, however in contrast to the above studies they found an evidence for a "remarkable variety of unfolding intermediates". 28

Solution and gas-phase behavior of lysozyme contrasts that of ubiquitin. It is approximately twice the size of ubiquitin with 129 amino acids and due to its four intramolecular disulfide bonds ${ }^{29}$, it often exists in highly stable folds both in solution and in the gasphase. ${ }^{30,31}$ In solution, the folding pathway of lysozyme is typically reported as a two-state transition between the native $\mathrm{N}$-state and the denatured helical $\mathrm{H}$-state without intermediate structures. However, kinetically trapped, partially folded transient intermediates may be possible. ${ }^{32}$ In the gas-phase, at ambient temperatures, the lysozyme $8+$ ion presents with a ${ }^{{ }^{D T}} \mathrm{CCS}_{\mathrm{He}}$ of $\sim 1350 \AA^{2}(\mathrm{~N}-$ state) whilst the unfolded $\mathrm{H}$-state has a ${ }^{\mathrm{DT}} \mathrm{CCS}_{\mathrm{He}}$ of $\sim 1750 \AA^{2} .{ }^{33}$

Here we employed a variable temperature ion mobility-mass spectrometer (VT IM-MS) ${ }^{34}$ in an attempt to record ion mobility spectra of the "unfolding intermediates", advocated by Breuker et al. For ubiquitin, our results indicate that the intermediates are initially highly elongated and then rapidly converge to compacted structures. We note that only the latter species are observed in ambient temperature IM experiments. The gas-phase folding pathway of non-reduced lysozyme is expectedly more restrained, resembling the two-state solution model. However, cryogenic IM-MS still captures some, partially folded intermediates between the $\mathrm{N}$ - and H-states.

Observation of these folding transitions and transient intermediates in well-studied proteins illustrates the potential of VT-IM-MS methodology in studying fast structural transitions, thus delineating their gas-phase un-folding pathways. The key advantage here 
is the ability to freeze the metastable transition states, assess their size and compare it to that of the "native" and "unfolded" forms.

\section{Experimental}

Samples. Ubiquitin (from bovine erythrocytes) and lysozyme (from chicken egg white) were purchased from Sigma Aldrich, UK. Ammonium acetate was purchased from Fisher Scientific, UK. Final protein concentrations were prepared to $50 \mu \mathrm{M}$ ubiquitin and $30 \mu \mathrm{M}$ lysozyme, both in $50 \mathrm{mM}$ ammonium acetate, $\mathrm{pH}$ 6.8 .

VT IM-MS. Our experimental arrangement and measurement principles have been described in detail elsewhere. ${ }^{34}$ Briefly, the ions are created in a nano-ESI source (capillary 1-1.4 kV), then transferred via two ion guides into the variable temperature (VT) IM cell. The temperature of the ion source and the first two guides is held close to $300 \mathrm{~K}$, whereas the temperature of the drift gas can be varied between 150 and $500 \mathrm{~K}$. Ion activation is induced by increasing the voltage offset between the first two ion guides, both held close to ambient temperature. In-source activated ions then enter the VT compartment. Inside the VT chamber, the ions acquire the temperature of the helium gas while being accumulated for 16 and $22 \mathrm{~ms}$ for ubiquitin and lysozyme, respectively. Upon release as $20 \mu$ s packets, the ions travel through the drift region $(50.5 \mathrm{~cm})$ where the mobility separation takes place. Compact structures drift faster than the extended forms of the same charge state. After IM separation, the $\mathrm{m} / \mathrm{z}$ of ions are measured in a time-of-flight mass analyzer. In such IM-MS experiments, m/z data obtained for each ion has an associated arrive time distribution (ATD), which can be then converted to a collision cross section distribution. ${ }^{34,35}$

\section{Ubiquitin}

We have obtained VT IM-MS data for the $5+$ and +6 charge states of ubiquitin with and without in-source activation. Figure 1 shows the collision cross section distributions obtained for $6+$ ubiquitin ions between 150 and $500 \mathrm{~K}$. The ${ }^{\mathrm{DT}} \mathrm{CCS}_{\mathrm{He}}$ distribution for the $5+$ and $7+$ charge states and the relevant mass spectra are presented in Figures S1-S3. Ion activation, prior to transfer into the variable temperature cell, occurs between the first and the second ion guides by increasing the voltage offset from 0 to $70 \mathrm{~V}$ (nonactivated (black) versus activated (dashed gray) traces in Figure 1). When the VT compartment is held at sub-ambient temperatures (lower 2 traces of Figure 1), the internal temperature of the collisionally activated ions is rapidly reduced; this may lead to kinetic trapping of energetic conformational states. Data obtained at sub-ambient temperatures indicates that these forms can be, to a large extent, preserved on the measurement timescale. When the IM cell is held at ambient or elevated temperatures, collisionally activated ions may re-fold during accumulation and, to a lesser extent during separation in the drift region. The typical drift times are between 4 and $12 \mathrm{~ms}$ and can be altered by changing the applied electric field. No apparent change is seen in these ${ }^{\mathrm{DT}} \mathrm{CCS}_{\mathrm{He}}$ distributions as a function of drift time, which implies that structural rearrangements occur primarily during accumulation.

At ambient temperature $(300 \mathrm{~K})$, non-activated ions present as a compact population around $950 \AA^{2}$, consistent with the reported literature data. ${ }^{19,36}$ Upon reducing the temperature, the ${ }^{\mathrm{DT}} \mathrm{CCS}_{\mathrm{He}}$ of the compact population shifts to $\sim 1050 \AA^{2}$ at $150 \mathrm{~K}$.

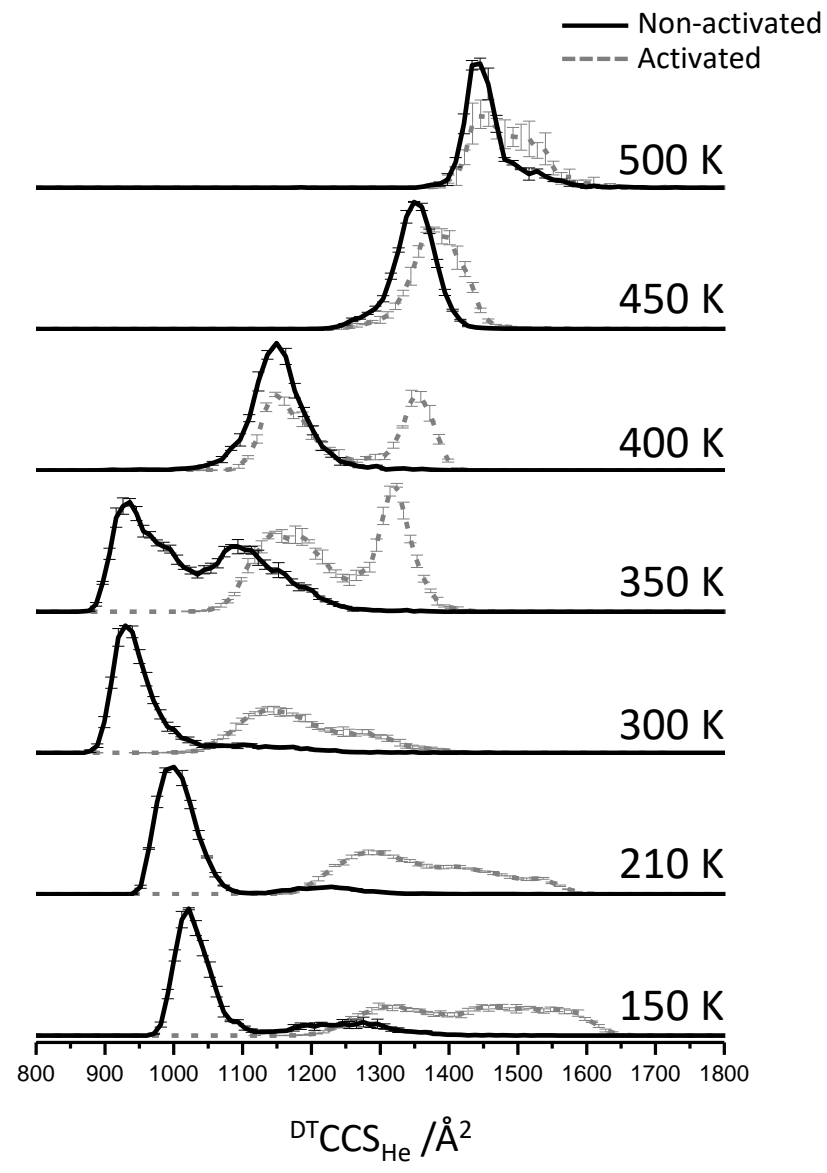

Figure 1. Collision Cross Section distributions of ubiquitin 6+ sprayed from $50 \mu \mathrm{M}$ solution in $50 \mathrm{mM}$ ammonium acetate, $\mathrm{pH}$ 6.8. Solid lines represent data obtained with no in-source activation at a range of temperatures. Dashed lines represent the respective data recorded with the in-source activation on. Error bars correspond to standard deviation from three, 1 minute long acquisitions. Activated data has been normalized to the area of the nonactivated data.

We attribute this increase to the expected dependence of long range ion-molecule interactions rather than a conformational transition. ${ }^{37}$ At $350 \mathrm{~K}$ and above, several conformational transitions are observed for non-activated ions. At room temperature, the ${ }^{\mathrm{DT}} \mathrm{CCS}_{\mathrm{He}}$ profiles of the activated ions appear between 1100 and $1400 \AA^{2}$. However, at $150 \mathrm{~K}$ source activation produces significantly more extended forms with ( ${ }^{\mathrm{DT}} \mathrm{CCS} \mathrm{S}_{\mathrm{He}} 1250$ - $1650 \AA^{2}$ ). Tuning the collisional activation conditions allows refolding intermediates to be captured in the low temperature drift tube (Figure S4). The most extended state achieved at $150 \mathrm{~K}\left(1650 \AA^{2}\right)$ aligns with the expected $150 \mathrm{~K}{ }^{\mathrm{DT}} \mathrm{CCS}_{\mathrm{He}}$ of the most extended state at $500 \mathrm{~K}$, suggesting this is a highly extended form; numerous other lower CCS intermediate states are also observed. Ion-molecule interactions may be different for elongated ions than for the compact forms, nevertheless the magnitude of the change and difference in the shape of the distribution cannot be explained solely by ionmolecule interaction potentials. ${ }^{38}$

\section{Lysozyme}

The above findings are in contrast with the data obtained from lysozyme. Figure 2 shows the ${ }^{\mathrm{DT}} \mathrm{CCS}_{\mathrm{He}}$ distributions for the $8+$ ions of lysozyme between 160 and $295 \mathrm{~K}$; the accompanying mass spectra and non-activated and activated data for 7+ ions can 
be found in the Figures S5 and S6. For the +8 ions, the nonactivated species (solid black line) predominantly present as the compact N-state $\left(\sim 1360 \AA^{2}\right.$ at $\left.295 \mathrm{~K}\right)$. As for ubiquitin, the ${ }^{\mathrm{DT} C \mathrm{C} \text { - }}$ $\mathrm{S}_{\mathrm{He}}$ increases with decreasing buffer gas temperature $(250 \mathrm{~K}$, $1370 \AA^{2} ; 210 \mathrm{~K}, 1400 \AA^{2} ; 160 \mathrm{~K}, 1460 \AA^{2}$ ) which again we attribute to the expected temperature dependence of the ion-molecule interaction. ${ }^{39}$ Theoretical ${ }^{\mathrm{DT}} \mathrm{CCS}_{\mathrm{He}}$ for 160 - $295 \mathrm{~K}$ were calculated using the projected superposition approximation (PSA) method. ${ }^{40}$ The change in theoretical ${ }^{\mathrm{DT}} \mathrm{CCS}_{\mathrm{He}}$ across the presented temperature range is predicted to be $\sim 6 \%$, in good agreement with that measured experimentally $(\sim 7 \%)$. Following collisional activation, we see an increase in the population of the extended state $\left(\sim 1750 \AA^{2}\right.$ at $\left.295 \mathrm{~K}\right)$ and the retention of some $\mathrm{N}$-state population. Following in-source activation at temperatures $295 \mathrm{~K}-250 \mathrm{~K}$, we observe partial refolding back to the $\mathrm{N}$-state (Figures 2 and S6). As we decrease the temperature of the drift cell further, we observe that population $\mathrm{H}$ shifts away from the un-activated $\mathrm{N}$-state, the effect being most pronounced at $160 \mathrm{~K}$. This indicates that as for ubiquitin, we are able to 'freeze' some of the transient intermediates within the timescale of the experiment. Similar behavior is found for the 7+ charge state (Figure S6), although the available activation energy is not sufficient to substantially populate the $\mathrm{H}$ state.

\section{Discussion}

For ubiquitin, we conclude that at low temperatures we are able to kinetically trap metastable intermediate/transition states which are not experimentally observable in ambient temperature measurements. At $350 \mathrm{~K}$ and above, the conformational profiles of the activated ions ubiquitin are significantly different to the low $\mathrm{T}$ profiles. Our data suggests that at elevated temperatures (above $150 \mathrm{~K})$, extended intermediates 'frozen out' at $150 \mathrm{~K}$ (1300-1600 $\AA^{2}$ ) can refold to adopt temperature specific conformations (e.g. $1150 \AA^{2}$ at $300 \mathrm{~K}$ ). In particular, at $400 \mathrm{~K}$ we observe two distinct populations, of apparently equal stability, both of which may be due to different annealing pathways from the extended forms. At $450 \mathrm{~K}$ and $500 \mathrm{~K}$, more extended forms dominate the ${ }^{\mathrm{DT}} \mathrm{CCS}_{\mathrm{He}}$ distributions which are also narrower, suggestive of a reduction in the number of conformations present. We can contemplate whether conformational transitions observed here bear any resemblance to the reversible conformational transitions, exhibited by ubiquitin in solution. ${ }^{13,22}$ Changes in ${ }^{\mathrm{DT}} \mathrm{CCS}_{\mathrm{He}}$ are to a large extent "reversible" for conformations between 1100 and $1650 \AA^{2}$ while this is not the case for the structures with ${ }^{{ }^{D T}} \mathrm{CCS}_{\mathrm{He}}$ below $1000 \AA^{2}$. We do not observe any "compact intermediates" which suggests that once activated, they cannot find the initial state in the timescale available. As an additional check, we performed in-source activation at a range of collision energies (Figure S4 and S7); yet again no "compact intermediates" are observed. Therefore, the initial compact conformers of ubiquitin $\left(\sim 950 \AA^{2}\right)$ are likely contain solution-phase specific secondary structural which can be preserved in the gas phase with very gentle ESI conditions. ${ }^{19}$ Ubiquitin data shows that all transitions from such solution-specific folds appear "irreversible" on the timescale of our experiment. This is in accord with the original idea of Smith and Light-Wahl: "Once the structure of the molecular ion has been lost, however, longrange coulombic forces should effectively preclude the reverse process. $" 41$

A contrasting behavior is observed for lysozyme where, even at $160 \mathrm{~K}$, there is a significant overlap of activated and non-activated arrival time distributions. This suggests that the structural motifs in activated ion populations are to a large extent similar to those in non-activated ions. This correlates with the known, high stability of lysozyme both in solution and in the gas-phase.

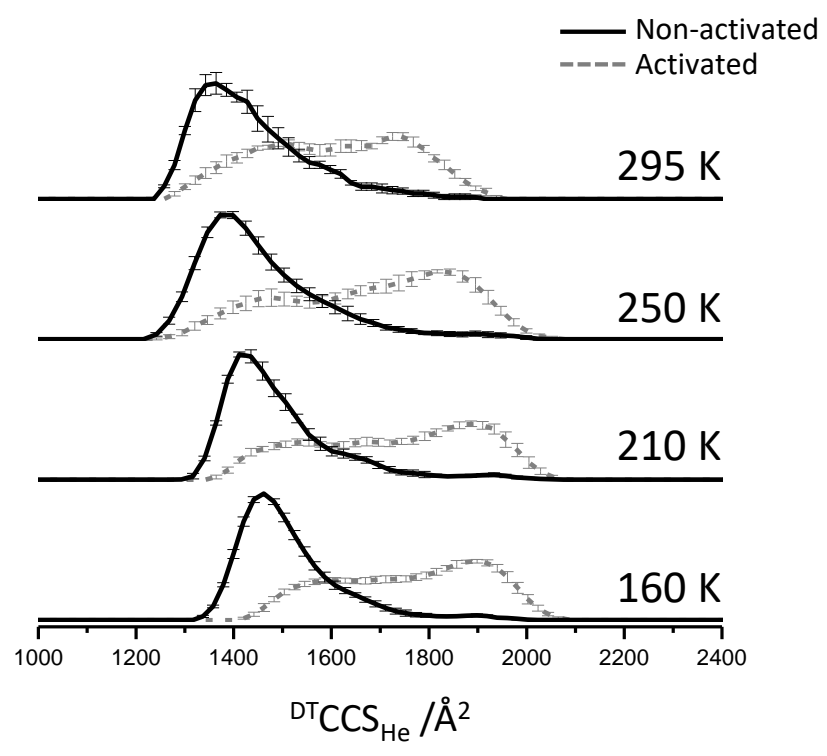

Figure 2. Collision cross section distributions of lysozyme $8+$ sprayed from $30 \mu \mathrm{M}$ solution in $50 \mathrm{mM}$ ammonium acetate, $\mathrm{pH}$ 6.8. Solid lines represent data obtained with no in-source activation at a range of temperatures (160 to $295 \mathrm{~K}$ ). Dashed lines represent the respective data recorded with in-source activation (voltage offset of $90 \mathrm{~V}$ ). Error bars correspond to standard deviation from six, 30 second long acquisitions. Activated data has been normalized to the area of the non-activated data.

The results obtained for both $5+$ charge state ubiquitin and 7+ charge state lysozyme (Figures S1 and S5, respectively) show effects considerably less pronounced than for the respective more highly charged species (Figures 1 and 2, respectively). The energy gained during the activation step is less for the lower charge states; moreover the drift time is longer and therefore the likelihood of rearrangement during IM separation is higher (quantitatively, the difference of either effect is approximately 17\%). Nevertheless, the magnitude of difference between +5 and +6 charge states for ubiquitin and +7 and +8 charge states for lysozyme suggests that the presence of one additional proton (or a lack of thereof) plays a key role during gas-phase unfolding and refolding of the protein. It has been shown previously that the coordination (self-solvation) plays an important role in this process. $^{25,26}$ To rule out the possibility of charge stripping during activation, mass selected IM-MS of ubiquitin $6+$ and $7+$ and lysozyme $8+$ and 9+ were recorded at increasing activation energies (see Figures S8A-B and S9A-B, respectively).

The above findings can be summarized with a toy picture of the gas-phase folding free energy landscapes compared to that in solution (Figure 3). Considering the data obtained for ubiquitin, a structurally flexible protein, we conclude that the transition from the initial "compact" ions (denoted $\mathrm{N}^{6+}, 950 \AA^{2}$ ) is irreversible on our experimental timescales - thus either the energy of this state is higher in the gas phase (solid line) or that the barrier between this and the intermediates and extended states is elevated in the absence of solution (dotted line). Following activation at room temperature, unfolding proceeds through a variety of intermediates and transition states (denoted TS) which at room temperature converge to structures at $1100-1400 \AA^{2}$. At cryogenic temperatures (blue shaded area), we can slow down the interconversion process and perhaps kinetically trap those metastable species, which results in broad CCS profile (Figure 1, 210-150K). We hypothesize that ubiquitin $\mathrm{N}^{6+}$ undergoes an inside-out transition, ${ }^{42}$ which may involve both self-solvating of "native 
protonation sites" and accommodating for the coulombic repulsions. In high temperature environment $(500 \mathrm{~K})$, the system presents as a single, substantially unfolded population at $\sim 1450 \AA^{2}$. Presence of a shoulder at $\sim 1530 \AA^{2}$ following activation, suggests presence of an additional high energy state.

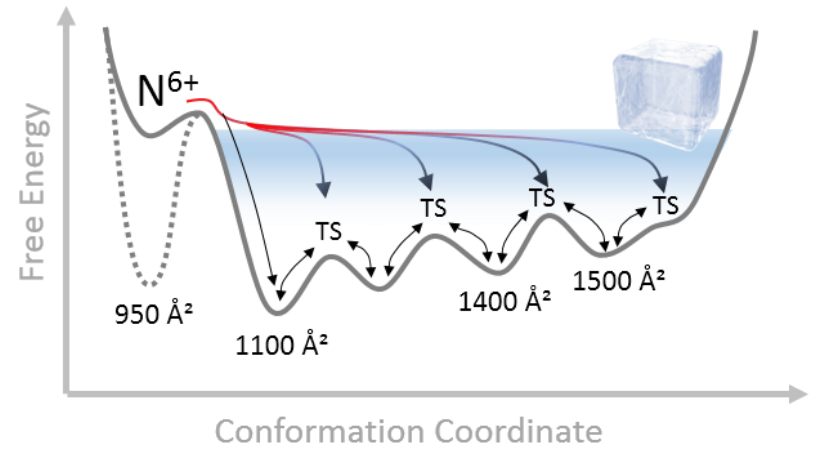

Figure 3. Hypothetical 1D gas-phase folding free energy surfaces of ubiquitin in the gas phase. The initial compact state obtained from solution via ESI process is denoted $\mathrm{N}^{6+}$. Upon activation ubiquitin can no longer access the native state, and samples a plethora of unfolded states via multiple transition states (denoted TS). Most of these transitions appear to be reversible. Shaded blue area depicts the principle of performing the measurements at low temperature interconverting and transition states can be preserved ('frozen') on the time scale of experiment. Corresponding sketches contrasting solution and gas phase behavior of ubiquitin and lysozyme are presented in Figure S10.

For Lysozyme, the conformational restriction induced by the disulfide bonding, prevents the protein from full departure from the native like fold. The structural diversity of the transient folding intermediates is, again, much decreased compared to that in the unrestricted protein. This suggests that solution and gas phase folding free energy surfaces for this conformationally restricted protein are somewhat similar (Figure S10).

For both charge states of lysozyme (Figures 2 and S6) the width of the un-activated ${ }^{\mathrm{DT}} \mathrm{CCSD}_{\mathrm{He}}$ at $250 \mathrm{~K}$ is greater than at $210 \mathrm{~K}$ and the data at 250 and $160 \mathrm{~K}$ indicates more resolved conformers at $\sim 1650 \AA^{2}$ as well as some $\mathrm{N}$-state. The enhanced resolution is expected - it should scale with $\sqrt{ } \mathrm{T} ;{ }^{43}$ the fact that we do not see this for ubiquitin provides an additional evidence that following transfer into the gas phase, $\mathrm{N}$-state conformers are readily disrupted by (unintentional) activation. The increased width at $250 \mathrm{~K}$ for un-activated lysozyme $8+$ has been reported by us previously ${ }^{33}$ and may indicate a temperature specific transition at around $0{ }^{\circ} \mathrm{C}$, which does not happen at lower temperatures.

\section{Summary and Outlook}

By manipulating solution and experimental conditions, it has been shown that gas-phase conformations of proteins can in turn be manipulated and transitions from compact to extended forms have been reported. Data and interpretation presented here attempts to expand the current understanding of gaseous, "native" conformations of proteins. Collectively, our results show that gas-phase unfolding can proceed through highly extended, intermediate states which then converge to "gas-phase compacted" structures. With our newly developed instrument and VT-IM-MS methodology, we can directly visualize the remarkable structural diversity of those states, first detected by ECD. ${ }^{28}$ By affecting the temperature at which the refolding occurs, we can direct the folding process of a protein. This highlights the potential of this technique to map free energy surfaces for proteins. In this work, we transpose our experimental findings to hypothetical free energy surfaces for the two contrasting cases.

Considering that a "compact" state of protein in the gas phase is itself a kinetically trapped form, for small and flexible proteins these measurements show that we still need to better understand the bias given to solvated ensembles by the electrospray and desolvation process and by the dominance of electrostatics in gasphase structures. In solution, a thermally induced, reversible conformational transition for ubiquitin has been reported to occur between 330 and $370 \mathrm{~K}$. Moreover, an irreversible transition was found to occur above $400 \mathrm{~K} .{ }^{13}$ Although it is difficult to compare the mechanistic details of the thermally induced unfolding in solution with that which occurs in the absence of solvent, our high temperature data is consistent with these solution-phase findings; however, it remains to be seen whether this is a general effect.

Finally, we note that the experiment presented here is analogous to "simulated annealing", an approach employed frequently in computational simulations of protein structure and where ubiquitin is often used as a benchmark. ${ }^{3}$ In the future we intend extend the VT-IM-MS method to allow for measurement of timescales of the folding events presented here. We envisage that such timeresolved data could constitute a unique tool for assessing protein dynamics in the gas phase.

\section{ASSOCIATED CONTENT}

\section{Supporting Information}

Details of experimental setup and data processing, ${ }^{\mathrm{DT}} \mathrm{CCS}_{\mathrm{He}}$ profiles of $5+$ and $7+$ ubiquitin and $7+$ lysozyme charge states, accompanying n-ESI mass spectra and data from ambient IM-MS control experiments. This material is available free of charge via the Internet at http://pubs.acs.org."

\section{AUTHOR INFORMATION}

\section{Corresponding Author}

*E-mail: perdita.barran@manchester.ac.uk

\section{Notes}

The authors declare no competing financial interests. JU and PEB devised the study. JJ ran initial experiments on lysozyme (data not shown), and RU BB, and FB performed subsequent experiments, and all analysis on lysozyme. JU drafted the manuscript with PEB and RU. All authors contributed to its final form.

\section{ACKNOWLEDGMENT}

Funding was provided by an EPSRC DTA award with CASE funding from Waters corp. for a PhD studentship for JU and by a BBSRC Industrial Case Studentship awarded to RU in collaboration with Covance Laboratories and instrumentation was supported by the BBSRC (awards: BB/Lo15048/1, $\mathrm{BB} / \mathrm{Ko17802/1}$ and $\mathrm{BB} / \mathrm{Ho1}_{3636 / 1}$ and the BBSRC/EPSRCfunded Manchester Synthetic Biology Research Centre, SYNBIOCHEM (BB/Mo17702/1)). We would like to thank Dr Rebecca Beveridge for reviewing the manuscript and useful comments and Professors Martin Jarrold and David Clemmer for inspiring us for the past 30 years. 


\section{REFERENCES}

(1) Jackson, S. E. Ubiquitin: a small protein folding paradigm. Org. Biomol. Chem. 2oo6, 4 (10), 1845-1853 DOI: 10.1039/b6oo829c.

(2) Jackson, S. E. How do small single-domain proteins fold? Fold. Des. 1998, 3 (4), 81-91 DOI: 10.1016/S13590278(98)00033-9.

(3) Piana, S.; Lindorff-Larsen, K.; Shaw, D. E. Atomiclevel description of ubiquitin folding. Proc. Natl. Acad. Sci. 2013, 110 (15), 5915-5920 DOI: $10.1073 /$ pnas.1218321110.

(4) Cornilescu, G.; Marquardt, J. L.; Ottiger, M.; Bax, a. Validation of protein structure from anisotropic carbonyl chemical shifts in a dilute liquid crystalline phase. J. Am. Chem. Soc. 1998, 120 (27), 6836-6837 DOI: 10.1021/ja9812610.

(5) Vijay-Kumar, S.; Bugg, C. E.; Wilkinson, K. D.; Cook, W. J. Three-dimensional structure of ubiquitin at 2.8 A resolution. Proc. Natl. Acad. Sci. U. S. A. 1985, 82, 3582-3585 DOI: 10.1073/pnas.82.11.3582.

(6) Stockman, B. J.; Euvrard, A.; Scahill, T. A. Heteronuclear three-dimensional NMR spectroscopy of a partially denatured protein: The A-state of human ubiquitin. J. Biomol. NMR 1993, 3, 285-296 DOI: 10.1007/BFoo212515.

(7) Schanda, P.; Forge, V.; Brutscher, B. Protein Folding and Unfolding Studied at Atomic Resolution by Fast Two-Dimensional NMR Spectroscopy. Proc. Natl. Acad. Sci. 2007, 104 (27), 11257-11262 DOI: 10.1073/pnas.0702069104.

(8) Chung, H. S.; Shandiz, A.; Sosnick, T. R.; Tokmakoff, A. Probing the Folding Transition State of Ubiquitin Mutants by Temperature-Jump-Induced Downhill Unfolding †. Biochemistry 2008, 13870-13877 DOI: DOI: 10.1021/bi8o16oze.

(9) Went, H. M.; Jackson, S. E. Ubiquitin folds through a highly polarized transition state. Protein Eng. Des. Sel. 2005, 18 (5), 229-237 DOI: 10.1093/protein/gzio25.

(10) Wilkinson, K. D.; Mayer, A. N. Alcohol-induced conformational changes of ubiquitin. Arch. Biochem. Biophys. 1986, 250 (2), 390-399 DOI: 10.1016/00039861(86)90741-1.

(11) Ibarra-Molero, B.; Loladze, V. V; Makhatadze, G. I.; Sanchez-Ruiz, J. M. Thermal versus guanidineinduced unfolding of ubiquitin. An analysis in terms of the contributions from charge-charge interactions to protein stability. Biochemistry 1999, 38 (25), 81388149 DOI: 10.1021/bi9905819.

(12) Kitahara, R.; Akasaka, K. Close identity of a pressurestabilized intermediate with a kinetic intermediate in protein folding. Proc. Natl. Acad. Sci. U. S. A. 2003, 100 (6), 3167-3172 DOI: 10.1073/pnas.063030910o.

(13) Wintrode, P. L.; Makhatadze, G. I.; Privalov, P. L. Thermodynamics of ubiquitin unfolding. Proteins Struct. Funct. Genet. 1994, 18 (3), 246-253 DOI: $10.1002 /$ prot.340180305.

(14) Mack, E. Average Cross-Sectional Areas of Molecules By. J. Am. Chem. Soc. 1924, 47 (1920), 2468-2482 DOI: 10.1021/jao1687aoo7.

(15) Jarrold, M. F. Peptides and Proteins in the Vapor Phase. Annu. Rev. Phys. Chem. 2ooo, 51, 179-207
DOI: 10.1146/annurev.physchem.51.1.179.

(16) Koeniger, S. L.; Clemmer, D. E. Resolution and Structural Transitions of Elongated States of Ubiquitin. J. Am. Soc. Mass Spectrom. 2007, 18 (2), 322-331 DOI: 10.1016/j.jasms.2006.09.025.

(17) Koeniger, S. L.; Merenbloom, S. I.; Sevugarajan, S.; Clemmer, D. E. Transfer of structural elements from compact to extended states in unsolvated ubiquitin. J. Am. Chem. Soc. 2006, 128 (35), 11713-11719 DOI: 10.1021/jao62137g.

(18) Myung, S.; Badman, E. R.; Lee, Y. J.; Clemmer, D. E. Structural Transitions of Electrosprayed Ubiquitin Ions Stored in an Ion Trap over $10 \mathrm{~ms}$ to $30 \mathrm{~s}$. J. Phys. Chem. A 2002, 106 (42), 9976-9982 DOI: 10.1021/jpo206368.

(19) Wyttenbach, T.; Bowers, M. T. Structural stability from solution to the gas phase: native solution structure of ubiquitin survives analysis in a solventfree ion mobility-mass spectrometry environment. $J$. Phys. Chem. B 2011, 115 (42), 12266-12275 DOI: 10.1021/jp206867a.

(20) Merenbloom, S. I.; Flick, T. G.; Daly, M. P.; Williams, E. R. Effects of select anions from the Hofmeister series on the gas-phase conformations of protein ions measured with traveling-wave ion mobility spectrometry/mass spectrometry. J. Am. Soc. Mass Spectrom. 2011, 22 (11), 1978-1990 DOI: 10.1007/s13361-011-0238-1.

(21) Zhao, Q.; Soyk, M. W.; Schieffer, G. M.; Fuhrer, K.; Gonin, M. M.; Houk, R. S.; Badman, E. R. An ion trap-ion mobility-time of flight mass spectrometer with three ion sources for ion/ion reactions. J. Am. Soc. Mass Spectrom. 2009, 20 (8), 1549-1561 DOI: 10.1016/j.jasms.2009.04.014.

(22) El-Baba, T. J.; Woodall, D. W.; Raab, S. A.; Fuller, D. R.; Laganowsky, A.; Russell, D. H.; Clemmer, D. E. Melting Proteins: Evidence for Multiple Stable Structures upon Thermal Denaturation of Native Ubiquitin from Ion Mobility Spectrometry-Mass Spectrometry Measurements. J. Am. Chem. Soc. 2017, 139 (18), 6306-6309 DOI: 10.1021/jacs.7bo2774.

(23) Clemmer, D. E.; Jarrold, M. F. Ion Mobility Measurements and Their Applications to Clusters and Biomolecules. J. Mass Spectrom. 1997, 32, 577$592 \quad$ DOI: 10.1002/(SICI)10969888(199706)32:6<577::AID-JMS530>3.0.CO;2-4.

(24) Dickinson, E. R.; Jurneczko, E.; Pacholarz, K. J.; Clarke, D. J.; Reeves, M.; Ball, K. L.; Hupp, T. R.; Campopiano, D. J.; Nikolova, P. V; Barran, P. E. Insights to the conformations of three structurally diverse proteins: Cytochrome c, p53 and MDM2, provided by Variable Temperature Ion Mobility Mass Spectrometry. Anal. Chem. 2015, 150128125604004 DOI: 10.1021/ac503720v.

(25) Valentine, S. J.; Counterman, A. E.; Clemmer, D. E. Conformer-Dependent Proton-Transfer Reactions of Ubiquitin Ions. J. Am. Soc. Mass Spectrom. 1997, 8, 954-961 DOI: 10.1016/S1044-0305(97)00085-8.

(26) Laszlo, K. J.; Munger, E. B.; Bush, M. F. Folding of Protein Ions in the Gas Phase after Cation-to-Anion Proton-Transfer Reactions. J. Am. Chem. Soc. 2016, 138 (30), 9581-9588 DOI: 10.1021/jacs.6bo4282.

(27) Schlierf, M.; Li, H.; Fernandez, J. M. The unfolding 
kinetics of ubiquitin captured with single-molecule force-clamp techniques. Proc. Natl. Acad. Sci. U. S. A.

2004, $101 \quad$ (19), $7299-7304$ DOI: 10.1073/pnas.0400033101.

(28) Breuker, K.; Oh, H.; Horn, D. M.; Cerda, B. A.; McLafferty, F. W. Detailed unfolding and folding of gaseous ubiquitin ions characterized by electron capture dissociation. J. Am. Chem. Soc. 2002, 124 (22), 6407-6420 DOI: 10.1021/jao12267j.

(29) Blake, C. C. F.; Koenig, D. F.; Mair, G. A.; North, A. C. T.; Phillips, D. C.; Sarma, V. R. Structure of hen eggwhite lysozyme: A three-dimensional Fourier synthesis at $2 \AA$ resolution. Nature 1965, 206 (4986), 757-761 DOI: 10.1038/206757ao.

(30) Schwalbe, H.; Grimshaw, S. B.; Spencer, A.; Buck, M.; Boyd, J.; Dobson, C. M.; Redfield, C.; Smith, L. J. A refined solution structure of hen lysozyme determined using residual dipolar coupling data. Protein Sci. 2001, 10 (4), 677-688 DOI: 10.1110/ps.43301.

(31) Valentine, S. J.; Anderson, J. G.; Ellington, A. D.; Clemmer, D. E. Disulfide-Intact and -Reduced Lysozyme in the Gas Phase: Conformations and Pathways of Folding and Unfolding. J. Phys. Chem. B 1997, 101 (19), 3891-3900 DOI: 10.1021/jp9702170.

(32) Kiefhaber, T. Kinetic traps in lysozyme folding. Proc. Natl. Acad. Sci. USA 1995, 92 (20), 9029-9033 DOI: 10.1073/pnas.92.20.9029.

(33) Berezovskaya, Y.; Porrini, M.; Barran, P. E. The effect of salt on the conformations of three model proteins is revealed by variable temperature ion mobility mass spectrometry. Int. J. Mass Spectrom. 2013, 345347, 8-18 DOI: 10.1016/j.ijms.2013.02.005.

(34) Ujma, J.; Giles, K.; Morris, M.; Barran, P. E.; Spectrometry, C. M. New High Resolution Ion Mobility Mass Spectrometer Capable of Measurements of Collision Cross Sections from 150 to 520 K. Anal. Chem. 2016, 88 (19), 9469-9478 DOI: 10.1021/acs.analchem.6bo1812.

(35) Pacholarz, K. J.; Barran, P. E. Distinguishing Loss of Structure from Subunit Dissociation for Protein Complexes with Variable Temperature Ion Mobility Mass Spectrometry. Anal. Chem. 2015, 87 (12) DOI: 10.1021/acs.analchem.5bo1063.

(36) Koeniger, S. L.; Merenbloom, S. I.; Clemmer, D. E. Evidence for Many Resolvable Structures within Conformation Types of Electrosprayed Ubiquitin Ions. J. Phys. Chem. B 2006, 110 (13), 7017-7021 DOI: 10.1021/jpo56165h.

(37) von Helden, G.; Wyttenbach, T.; Bowers, M. T. Inclusion of a MALDI ion source in the ion chromatography technique: conformational information on polymer and biomolecular ions. Int. J. Mass Spectrom. Ion Process. 1995, 146-147, 349-364 DOI: 10.1016/o168-1176(95)04211-3.

(38) Bleiholder, C.; Wyttenbach, T.; Bowers, M. T. A novel projection approximation algorithm for the fast and accurate computation of molecular collision cross sections (I). Method. Int. J. Mass Spectrom. 2011, 308 (1), 1-10 DOI: 10.1016/j.ijms.2011.06.014.

(39) Wyttenbach, T.; Von Helden, G.; Batka, J. J.; Carlat, D.; Bowers, M. T. Effect of the long-range potential on ion mobility measurements. J. Am. Soc. Mass Spectrom. 1997, 8 (3), 275-282 DOI: 10.1016/S10440305(96)00236-X.

(40) Wyttenbach, T.; Bleiholder, C.; Bowers, M. T. Factors contributing to the collision cross section of polyatomic ions in the kilodalton to gigadalton range: application to ion mobility measurements. Anal. Chem. 2013, 85 (4), 2191-2199 DOI: 10.1021/ac3029008.

(41) Smith, R. D.; Light-Wahl, K. J. The observation of non-covalent interactions in solution by electrospray ionization mass spectrometry: Promise, pitfalls and prognosis. Biol. Mass Spectrom. 1993, 22 (9), 493-501 DOI: 10.1002/bms.1200220902.

(42) Meyer, T.; Gabelica, V.; Grubmüller, H.; Orozco, M. Proteins in the gas phase. WIREs Comput. Mol. Sci. 2013, 3 (4), 408-425 DOI: 10.1002/wcms.1130.

(43) Rokushika, S.; Hatano, H.; Bairn, M. A.; Hill, H. H. Resolution Measurement for Ion Mobility Spectrometry. Anal. Chem. 1985, 57, 1902-1907 DOI: 10.1021/acoo286ao23. 


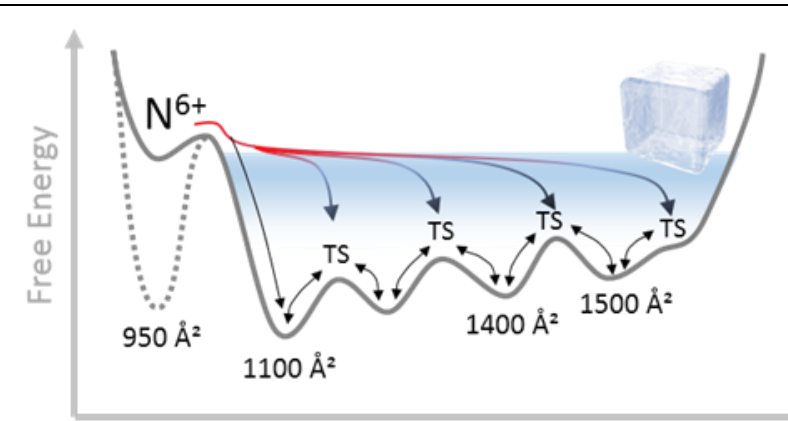

Conformation Coordinate

TOC Graphic 